\title{
Carbon Transport and Escape Fraction in a High Density Plasma Beam (P1-096)
}

\author{
G.A. van Swaaija,*, K. Bystrov ${ }^{\mathrm{a}}$, D. Borodin ${ }^{\mathrm{b}}$, A. Kirschner ${ }^{\mathrm{b}}$, T. Zaharia ${ }^{\mathrm{a}}$, \\ L.B. van der Vegt ${ }^{\mathrm{a}}$, G. De Temmerman ${ }^{\mathrm{a}}$, W.J. Goedheer ${ }^{\mathrm{a}}$ \\ ${ }^{a}$ FOM Institute DIFFER - Dutch Institute for Fundamental Energy Research, Association \\ EURATOM-FOM, Partner in the Trilateral Euregio Cluster, PO. Box 1207, 3430 BE \\ Nieuwegein, the Netherlands \\ ${ }^{b}$ Institute for Energy and Climate Research - Plasma Physics, Forschungszentrum Jülich \\ GmbH, Association EURATOM-FZJ, Partner in the Trilateral Euregio Cluster, Jülich, \\ Germany
}

\begin{abstract}
Hydrocarbon injection experiments on molybdenum targets facing high-density plasmas in Pilot-PSI were simulated with the 3D Monte Carlo impurity transport and PSI code ERO. Impurity transport and calculation of redeposition profiles were decoupled by calculating carbon redistribution matrices with ERO. Redeposition was found to be strongly dependent on the electron density. The calculated average number of recycling events of hydrocarbon molecules on the surface went up from from 1.5 for $\mathrm{n}_{e}=5 \cdot 10^{19} \mathrm{~m}^{-3}$ to 19.2 for $\mathrm{n}_{e}=4 \cdot 10^{20} \mathrm{~m}^{-3}$; at the latter density, only $2.4 \%$ of the hydrocarbon molecules escapes the simulated plasma beam without returning to the target at least once. Agreement with experimental deposition profiles in argon was fair. The results in hydrogen point towards a strong gradient in chemical erosion yield along the target.

Keywords:

PACS: 28.52.Fa; 52.40.Hf; 52.25.Vy; 52.65.Pp. PSI-20 keywords: Monte Carlo simulation, hydrocarbon layers, chemical erosion, redeposition.
\end{abstract}

\section{Introduction}

Carbon is an important plasma-facing material (PFM) for the walls, limiters, and divertors of tokamaks. However, under high hydrogen fluxes it suffers from severe chemical erosion, even at low ion energies. Hydrogen atoms and ions react with the carbon wall material and form various hydrocarbon molecules

\footnotetext{
${ }^{*}$ Corresponding author, presenting author

Email address: g.a.vanswaaij@differ.nl (G.A. van Swaaij)
} 
such as $\mathrm{C}(\mathrm{H}, \mathrm{D}, \mathrm{T})_{4}$. These hydrocarbon molecules get ionised in the plasma, and are subsequently transported back to the wall where they can redeposit. Such carbon redeposition can be either beneficial or harmful, depending on the site of redeposition. If the carbon redeposits in a remote area where the hydrogenic flux density is low, thick redeposits are formed which can retain large amounts of radioactive tritium. If ITER will use a carbon divertor, this co-deposition problem is predicted $(1,2)$ to be a major limiting factor in the duration of tritium campaigns. In contrast, redeposition of carbon close to the site of erosion reduces the net erosion rate of the divertor surface, and is therefore actually desirable. High electron densities, such as expected in front of the ITER divertor, will cause significant chemical erosion. But simultaneously, a high electron density will also lead to increasingly local redeposition due to the decreasing mean free path for ionization. Understanding of the balance between increasing net erosion and increasing local redeposition is clearly of utmost importance.

In this study, experiments in the high-density linear plasma generator PilotPSI were analysed using the 3D Monte Carlo impurity tracing PSI code ERO $(3,4)$. Detailed experimental results are reported elsewhere in these proceedings (5). Pilot-PSI is capable of producing ITER-divertor relevant plasma beams; plasma parameters in the range $B=0.4-1.6 \mathrm{~T}, \mathrm{n}_{e} \leq 10^{21} \mathrm{~m}^{-3}$, and $\mathrm{T}_{e}=0.5$ $3 \mathrm{eV}$ can be achieved. A water-cooled molybdenum plasma-facing target was used. Molybdenum is not sputtered in Pilot-PSI because of the low ion energies $(2-5 \mathrm{eV})$. Methane was injected through a hole in the center of the target; this is aligned with the center of the plasma beam. The full-width at half maximum (FWHM) of the $\mathrm{n}_{e}$ profile was $12 \mathrm{~mm}$.

Here we investigate which fraction of hydrocarbons escapes the plasma, how often they return to the target before escaping the plasma, and whether we 
can explain the observed deposition profiles. Concurrent deposition and reerosion complicate analysis of targets exposed to hydrogen plasma. Therefore, experiments and analysis were also performed in argon plasma, in which there is no chemical erosion of the carbon deposits.

\section{Simulation methodology}

In the ERO simulations, methane test particles enter the plasma at a thermal energy distribution corresponding to a source temperature of $800{ }^{\circ} \mathrm{C}$ (approximately the target temperature), with a cosine angular distribution. For simplicity, it was assumed that both externally injected hydrocarbon molecules and reflected molecules and the various chemically eroded hydrocarbon species each enter the plasma with those energy and angular distributions. This allowed us to greatly simplify the analysis: it is now possible to separate the modeling of impurity transport in the plasma and the calculation of actual redeposition profiles on the target. This method will be described in section 4 .

After entering the plasma, the molecules get ionised and dissociated predominantly through charge exchange with plasma ions and dissociative recombination with electrons. Reaction rates for methane breakdown come from (6). In addition to those rates, charge exchange reactions with argon were included (see section 3). The modeling used a static background plasma, with plasma parameters from experimental Thomson scattering measurements. To verify the applicability of the modeling assumptions in Pilot-PSI conditions, experimental and simulated photon emission plumes have been successfully compared; those results were reported elsewhere (7).

\section{Experiment}

A detailed overview of experimental results can be found elsewhere in these proceedings (5); they will be summarised here. 
$\mathrm{CH}_{4}$ was injected into the center of plasma beams in Pilot-PSI, through a hole in molybdenum samples. The plasma beam strikes the sample at a perpendicular angle. Both hydrogen and argon plasmas were used. The molybdenum sample is not sputtered. The $\mathrm{CH}_{4}$ injection rate was $3 \mathrm{sccm}\left(1 \mathrm{sccm} \equiv 4.42 \cdot 10^{17}\right.$ molecules/s). Each target was exposed for $100 \mathrm{~s}$.

The injected methane always formed deposits; the structure of those deposits depended primarily on the surface temperature. With $500 \mathrm{~K}<\mathrm{T}_{\text {surface }}<700$ $\mathrm{K}$, cauliflower-like microparticles were found, embedded in an $a-\mathrm{C}: \mathrm{H}$ layer. With $\mathrm{T}_{\text {surface }}>800 \mathrm{~K}$, no $a-\mathrm{C}: \mathrm{H}$ layer but only cauliflower-like particles were found. Because of the strong uncertainties in the growth rate of those particles, those samples were not further analysed with simulations.

On samples with surface temperatures below $500 \mathrm{~K}, a-\mathrm{C}: \mathrm{H}$ films were formed. Photographs of two such samples are shown in figure 1. The films cover the entire surface of the sample, even though they partially flaked off in the center after exposure to air. Redeposition rates of these films were measured with a mass balance, and redeposition patterns were measured with spectroscopic ellipsometry. Results are presented in section 5 .

\section{Inclusion of argon plasmas in ERO}

In the plasmas under consideration, with $\mathrm{T}_{e}<5 \mathrm{eV}$, the primary ionisation mechanism of hydrocarbons is charge exchange with plasma ions. Simulations in argon plasmas therefore require taking into account charge exchange reactions of the form $\mathrm{Ar}^{+}+\mathrm{CH}_{x} \rightarrow \mathrm{Ar}+\mathrm{CH}_{y}^{+}\left(+\mathrm{H}, \mathrm{H}_{2}\right)$. All other relevant reactions in the hydrocarbon break-up are electron-impact reactions, of which the rates of course do not depend on the plasma element (hydrogen or argon). Those reaction rates were taken from (6).

Energy-resolved reaction rates and branching ratios for the reaction 
$\mathrm{Ar}^{+}+\mathrm{CH}_{4} \rightarrow$ products were taken from (8), which has rate constants for the energy range from $5 \cdot 10^{-4}$ to $4 \mathrm{eV}$. The total rate constant given in the energy range under consideration is $1.41 \cdot 10^{-9} \cdot(\mathrm{E}[\mathrm{eV}])^{0.35}\left[\mathrm{~m}^{-3} \mathrm{~s}^{-1}\right]$. This is significantly slower than the charge exchange reaction between $\mathrm{H}^{+}$and $\mathrm{CH}_{4}$. We thus expect the penetration depth of neutral hydrocarbon molecules to be significantly larger in argon plasma than in a hydrogen plasma with comparable electron density.

One particularity of the charge exchange reaction between argon ions and methane is that the reaction does not produce $\mathrm{CH}_{4}^{+}$ions in the energy range under consideration. Rather, product ions are $\mathrm{CH}_{3}^{+}(85 \pm 3 \%)$ and $\mathrm{CH}_{2}^{+}(15 \pm 3 \%)$. The primary hydrocarbon recombination channel is dissociative recombination

of the form $e^{-}+\mathrm{CH}_{x} \rightarrow \mathrm{CH}_{x-y}+\left(\mathrm{H}, \mathrm{H}_{2}\right)$. Thus, the absence of $\mathrm{CH}_{4}^{+}$ions also implies an absence of $\mathrm{CH}_{3}$ radicals: those would have to be formed by the dissociative recombination of $\mathrm{CH}_{4}^{+}$. Because there are no $\mathrm{CH}_{3}$ radicals, the only other charge exchange reactions that play a role are those involving $\mathrm{CH}_{2}+\mathrm{Ar}^{+}$ and $\mathrm{CH}+\mathrm{Ar}^{+}$. Unfortunately, there are no reaction rates available in the literature for these secondary reactions. Therfore the measured reaction rates and branching ratios for the charge exchange of $\mathrm{CH}_{4}$ with argon have been used as a first approximation for those rates.

\section{Impurity redistribution matrices}

ERO was used to simulate transport of hydrocarbon molecules through the plasma. The calculation of layer growth was then done in post-processing. The method that was used for this is very similar to the one applied in (9) and (10). It will be summarised briefly here.

The modeled sample surface was divided in 15 concentric annular bins with widths of $1 \mathrm{~mm}$ each, with the injection nozzle in the center of the sample. 
We now consider the probability that a hydrocarbon molecule $C H_{x}$ that leaves the surface from bin $i$, will return as $\mathrm{CH}_{y}$ or $\mathrm{CH}_{y}^{(+)}$to surface bin $j$. This probability we call $\mathrm{M}_{i j}^{C H_{x} \rightarrow C H_{y}}$. Since we consider 15 different surface bins and 5 different hydrocarbon species $\left(\mathrm{CH}_{4}, \mathrm{CH}_{3} \cdots \mathrm{C}\right), \mathrm{M}$ has $5^{2} \cdot 15^{2}$ elements. The complete matrix $\mathrm{M}$ is called the hydrocarbon redistribution matrix. This matrix is completely independent of parameters such as the reflection probability of the various hydrocarbon molecules, the erosion yield, and the injection rate; it only depends on the plasma transport. Therefore, the calculation of the redistribution matrix and the subsequent PSI modeling can be separated.

To calculate $\mathrm{M}_{i j}^{C H_{x} \rightarrow C H_{y}}$ for given plasma conditions, sets of $5 \cdot 15$ ERO simulations were be performed (as we consider 5 different hydrocarbon species and 15 surface bins). 5,000 test particles were followed in every run. This gave sufficient statistics to compile the redistribution matrices.

After obtaining M, deposition profiles were calculated. In the experiments, $\mathrm{CH}_{4}$ was injected through a hole in the center of the target (at surface bin 0 ) with injection rate $\Gamma_{\text {source }}$. This leads to influxes of various hydrocarbon species $\mathrm{CH}_{x}$ at every bin $i$ :

$$
\Gamma_{i}^{\mathrm{CH}_{\mathrm{x}}}=\Gamma_{\text {source }} \cdot \mathrm{M}_{0 i}^{C H_{4} \rightarrow C H_{x}}
$$

Only part of this is deposited:

$$
\Gamma_{i}^{\mathrm{DEP}}=\sum_{x} \Gamma_{i}^{\mathrm{CH}} \cdot S_{\mathrm{CH}_{\mathrm{x}}}
$$

with $S_{\mathrm{CH}_{\mathrm{x}}}$ the sticking probability per species, and the summation running over all hydrocarbon molecules under consideration. The rest of the returning carbon is reflected, and redistributed over the target again. Of course, hydrocarbon molecules can be reflected multiple times; the calculation takes this into account numerically by following particles up to a maximum of 100 reflections, after 
which only a negligible fraction of the injected hydrocarbon remains.

After one calculation step, a layer of carbon is formed on the target. In $\mathrm{H}$ plasma, this layer is chemically eroded due to the incoming ion flux. The eroded carbon flux is the product of the ion flux $\Gamma^{H^{+}}$and erosion yield $Y$. If this product is larger than the amount of carbon remaining in the layer, then the entire layer is removed. The eroded carbon gets redistributed over the target in the same way as the externally injected hydrocarbon. This procedure is numerically repeated until the deposition rate converges along the target. In Ar plasma, there is no erosion, because chemical sputtering does not occur and the ion energy in Pilot-PSI $(<10 \mathrm{eV})$ is not sufficient to physically sputter the carbon layer.

The sticking probability $S_{\mathrm{CH}_{\mathrm{x}}}$ of every hydrocarbon radical was taken from MD simulations (11). In principle, the sticking probability is dependent on the ion energy. The hydrogen ions are accelerated in the pre-sheath towards the sound velocity, and their ion energy is determined by the sound speed plus the sheath potential drop (around $2-3 \cdot k_{B} T_{e}$ ). However, $\mathrm{CH}_{4}^{+}$ions are 16 times heavier than the hydrogen ions. After ionisation they thermalise quite efficiently with the hydrogen plasma due to the efficiency of Coulomb collisions. This means that the heavy $\mathrm{CH}_{x}^{+}$ions approach the target with speeds close to the sound speed of the background hydrogen plasma. Due to their greater mass, their energy is then much higher than the hydrogen ion energy. In $T_{e}=1 \mathrm{eV}$, $\mathrm{n}_{e}=10^{20} \mathrm{~m}^{-3}$ plasma, the average ion energy of all simulated hydrocarbon impurities (neutral and ionic) at the target is $11.0 \mathrm{eV}$, with a standard deviation of $7.8 \mathrm{eV}$. MD sticking probabilities corresponding to these energies are: $90 \%$ for $\mathrm{C}$ and $\mathrm{CH}, 85 \%$ for $\mathrm{CH}_{2}, 60 \%$ for $\mathrm{CH}_{3}$, and $5 \%$ for $\mathrm{CH}_{4}$. Those sticking rates were used throughout this study. 


\section{Results}

The total redistribution of $\mathrm{CH}_{4}$ is shown in figure 2 for two different plasma conditions: hydrogen plasma with $\mathrm{n}_{e}=2 \cdot 10^{20} \mathrm{~m}^{-3}$ and an argon plasma with $\mathrm{n}_{e}=5.5 \cdot 10^{20} \mathrm{~m}^{-3}$. In both plasmas, the matrices are strongly peaked around the diagonal with $r_{\text {destination }}=r_{\text {start }}$. This indicates that redeposition is quite localised: hydrocarbon molecules tend to get deposited close to the site of erosion.

\subsection{Hydrocarbon recycling, and importance of electron density}

The cause of the strongly localised redeposition seen from figure 2 is the short ionization mean free path $\lambda_{m f p}$ of $\mathrm{CH}_{4}$ in plasmas. For $0.3 \mathrm{eV}<\mathrm{T}_{e}<$ $2 \mathrm{eV}$, the rate coefficient of the primary ionization mechanism (charge exchange with $\mathrm{H}^{+}$ions $)$is $(8 \pm 2) \cdot 10^{-15} \mathrm{~m}^{-3}$. Assuming that the methane enters the plasma with a thermal velocity distribution around $800 \mathrm{~K}$, we now find $\lambda_{m f p}=$ $\left\langle v_{\mathrm{CH}_{x}}\right\rangle /\left(\mathrm{n}_{i} \cdot\langle\sigma v\rangle_{\mathrm{CX}}\right)=3.2 \mathrm{~mm}$ at $\mathrm{n}_{e}=1 \cdot 10^{20} \mathrm{~m}^{-3}$, and $\lambda_{m f p}=0.8 \mathrm{~mm}$ at $\mathrm{n}_{e}=4 \cdot 10^{20} \mathrm{~m}^{-3}$.

Indeed, in our calculations the fraction of injected $\mathrm{CH}_{4}$ that escapes the plasma beam without returning to the sample is a strong function of $\mathrm{n}_{e}$. At $\mathrm{n}_{e}=4 \cdot 10^{20} \mathrm{~m}^{-3}$, only $2.4 \%$ escapes the plasma without at last striking the sample once. In plasma with $\mathrm{n}_{e}=1 \cdot 10^{20} \mathrm{~m}^{-3}$ this fraction increases to $19 \%$. The full width at half maximum of the beam's Gaussian $\mathrm{n}_{e}$ profile was $12 \mathrm{~mm}$ in all cases.

Of course, if the carbon erosion is strong enough, eventually all the injected methane will escape the plasma beam after a number of reflections and/or cycles of deposition followed by re-erosion. We now have the total redistribution matrix. Using it, we can calculate the average number of times that a hydrocarbon molecule thus visits the surface before it escapes the plasma beam. To 


\begin{tabular}{c|c|c|c} 
Gas & $n_{e}\left(\mathbf{m}^{-3}\right)$ & $T_{e}(\mathbf{e V})$ & Redep. rate $\mathbf{( \% )}$ \\
\hline hydrogen & $1.8 \cdot 10^{20}$ & 2.2 & $14 \pm 5$ \\
hydrogen & $1.6 \cdot 10^{20}$ & 0.3 & $21 \pm 8$ \\
hydrogen & $2 \cdot 10^{20}$ & 0.3 & $38 \pm 8$ \\
argon & $5.5 \cdot 10^{20}$ & 1.3 & $54 \pm 22$
\end{tabular}

Table 1: Redeposition rates, determined from mass gain.

obtain this value, the erosion yield was set to infinity, and statistics were gathered on the number of surface visits. A histogram, along with the averages, is shown in figure 3. In higher-density plasmas, methane molecules visit the sample frequently, up to an average of 19.2 visits for $\mathrm{n}_{e}=4 \cdot 10^{20}$. This shows that recycling of hydrocarbon molecules is quite strong; it is a major factor in the interpretation of erosion/redeposition experiments in these plasmas.

\subsection{Erosion yield and layer growth}

Deposited layer thicknesses at the outside of the sample were measured by spectroscopic ellipsometry; measurements close to the center of the sample were not successful due to the flaking of the layers. Error bars of up to $50 \%$ in the layer thickness were necessary due to the inhomogeneity of the layer thickness and the surface roughness of the Mo sample. The layer density was found to be $0.8 \mathrm{~g} \mathrm{~cm}^{-3}$ by comparing the refractive index to tabulated values (12). By using this layer density, deposition profiles from simulation can be compared with experimental results.

In order to find which value of the erosion yield best matches experiment, runs were performed using different values of the erosion yield. Experimental layer thicknesses are shown for both argon and hydrogen plasmas in figure 4, along with simulated redeposition profiles. The total redeposition rates, as determined from mass gain measurements, are shown in table 1.

The calculated total redeposited fraction in argon was $65.0 \%$; this is within the experimental error bar. The redeposition profile does not match exactly, 
but simulation and experiment are in the same order of magnitude.

The redeposition profiles on the edge of the samples exposed to hydrogen plasma are best fitted by assuming an erosion yield $Y=0.2 \%$. Assuming $Y>0.5 \%$ leads to complete suppression of film growth on the periphery of the sample. However, with $Y=0.2 \%$, a large redeposited fraction of $84.8 \%$ is calculated, due to the strongly local redeposition. This greatly exceeds the experimental redeposition efficiency. A yield $5 \%$ results in a redeposition efficiency of $31.7 \%$, which is much closer to experiment. However, calculations with that erosion yield predict no film growth in the periphery of the sample; this does not match the experiment.

The discrepancy between those results suggests that it is not reasonable to assume a constant erosion yield across the sample. This is not necessarily surprising. The value of the chemical erosion yield of carbon deposits facing high flux hydrogen plasma is dependent on many factors, for example the flux density and ion energy (13), surface temperature (14), surface roughness (15), hydrogen content of the layer, and $s p^{2} / s p^{3}$ hybridisation. For temperatures below $650 \mathrm{~K}$ the erosion yield tends to increase quite strongly with surface temperature. In Pilot-PSI, the temperature on the inside of the sample is consistently higher than on the periphery due to the higher heat flux. This can cause the erosion yield profile to be maximal in the center of the sample. Indeed, on the periphery of samples with $\mathrm{T}_{\text {surface }}>800 \mathrm{~K}$, no film growth was observed, and the total redeposition efficiency was very low $(<10 \%)$. This result, too, confirms a strong dependence of the chemical erosion yield of $a-\mathrm{C}: \mathrm{H}$ on the surface temperature profile. 


\section{Acknowledgments}

This work, supported by the European Communities under the contract of Association between EURATOM/FOM, was carried out within the framework of the European Fusion Programme with financial support from NWO. The views and opinions expressed herein do not necessarily reflect those of the European Commission.

\section{References}

[1] G. Federici, et al., J. Nucl. Mater. 313-316 (2003) 11-22.

[2] A. Kirschner, et al., J. Nucl. Mater. 390-391 (2009) 152-155.

[3] A. Kirschner, V. Philipps, J. Winter, U. Koegler, Nucl. Fusion 40 (2000) 989.

[4] D. Borodin, et al., Contrib. Plasma Phys. 50 (2010) 432-438.

[5] K. Bystrov, et al., 2012. These proceedings.

[6] R. Janev, D. Reiter, Phys. Plasmas 9 (2002).

[7] G. van Swaaij, et al., 2012. Submitted.

[8] P. Tosi, et al., J. Phys. Chem. 99 (1995) 15538-15543.

[9] K. Schmid, Nucl. Fusion 48 (2008) 105004.

[10] K. Schmid, J. Nucl. Mater. 415 (2011) S284-S288.

[11] K. Tichmann, U. von Toussaint, W. Jacob, J. Nucl. Mater. 420 (2011) 291-296.

[12] W. Jacob, Thin Solid Films 326 (1998) 1-42. 
[13] J. Roth, et al., J. Nucl. Mater. 337-339 (2005) 970-974.

[14] J. Roth, J. Nucl. Mater. 266-269 (1999) 51-57.

[15] Y. Ueda, et al., J. Nucl. Mater 390-391 (2009) 44-48. 


\section{Figures}

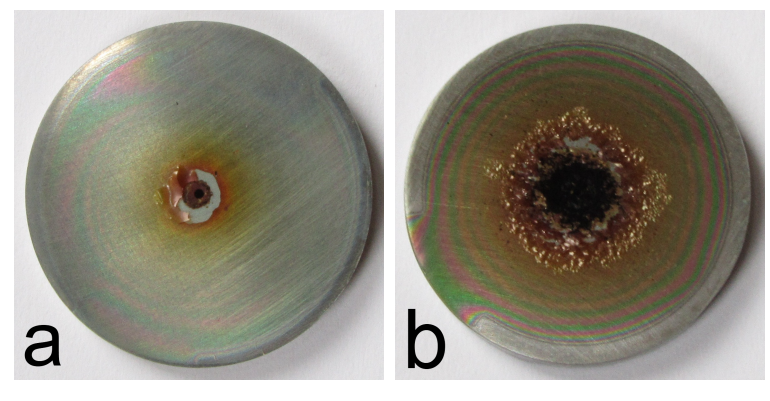

Figure 1: Molybdenum samples after methane injection experiments. Target a was exposed to hydrogen plasma $\left(\mathrm{T}_{e}=0.3 \mathrm{eV}, \mathrm{n}_{e}=1.6 \cdot 10^{20} \mathrm{~m}^{-3}\right)$. Target $\mathrm{b}$ was exposed to argon plasma $\left(\mathrm{T}_{e}=1.3 \mathrm{eV}, \mathrm{n}_{e}=5.5 \cdot 10^{20} \mathrm{~m}^{-3}\right)$. Both samples are completely covered with polymer-like $a$-C:H film. Flaking of the deposits occurred after exposure to the atmosphere. The diameter of the samples is $25 \mathrm{~mm}$. 


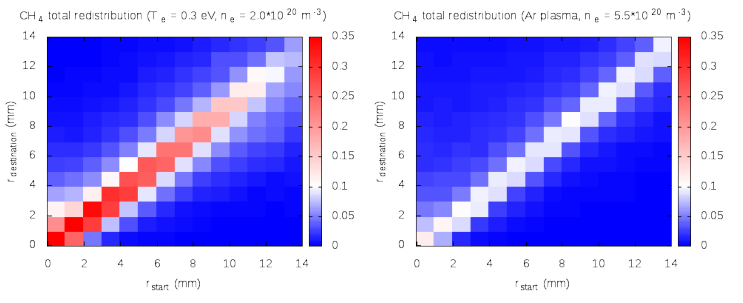

Figure 2: Total redistribution of $\mathrm{CH}_{4}$ in typical plasmas: hydrogen with $\mathrm{n}_{e}=2 \cdot 10^{20} \mathrm{~m}^{-3}$ and argon with $\mathrm{n}_{e}=5.5 \cdot 10^{20} \mathrm{~m}^{-3}$. The plots show $\sum_{x=\{0 \cdots 4\}} M^{C H_{4} \rightarrow C H_{x}}$. 


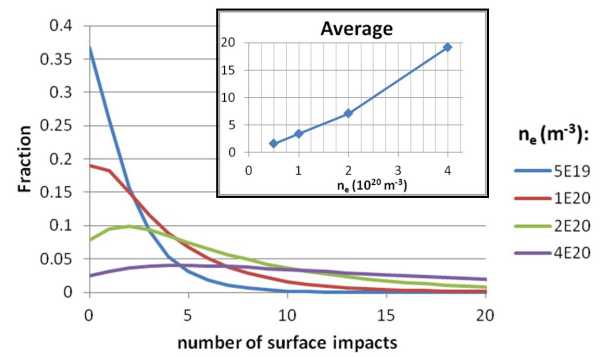

Figure 3: Distribution function of the number of surface visits before escaping the plasma beam, at various densities. Inset shows averages. 


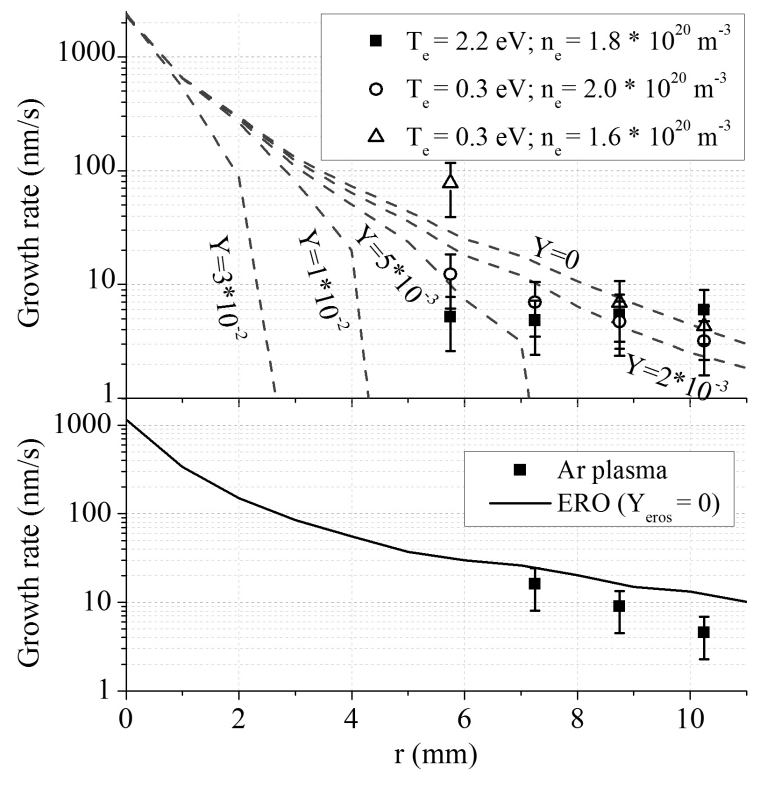

Figure 4: Deposition profiles measured by spectroscopic ellopsometry (points) together with results from ERO (curves), in both hydrogen (upper picture) and argon (lower picture) plasma. Growth rates in the center of the samples could not be accurately measured due to flaking of the layers. The plasma parameters used for the ERO calculations correspond those facing the samples in figure 1. In hydrogen, various simulation runs were made with different values assumed for the chemical erosion yield Y. 